\section{HPV16-DNA-Methylierung: Prädiktor für CIN2+}

Eine persistierende HPV16-Infektion ist Hauptursache für Zervixkarzinome, doch führen die Infektionen nur bei wenigen Frauen tatsächlich zu Neoplasien. Liefern DNA-Methylierungsmuster Hinweise auf eine drohende neoplastische Entwicklung?

$\mathrm{D}$ ie DNA-Methylierung des Humanen Papillomavirus Typ 16 (HPV16) ist einigen Untersuchungen zufolge mit einem erhöhten Risiko für Präkanzerosen, z. B. einer zervikalen intraepithelialen Neoplasie vom Grad 2 oder schlechter (CIN2+) assoziiert. Im Rahmen der longitudinalen Guanacaste/Costa RicaKohortenstudie wurde die Assoziation zwischen HPV16-DNA-Methylierung und Alter, Viruslast, viraler Persistenz sowie dem CIN2+-Risiko der Probandinnen untersucht. Ausgewählt wurden 273 Zervix-Gewebeproben (Proben von diagnostizierten Frauen und Proben von noch nicht positiv diagnostizierten Frauen = prädiagnostische Proben). 92 Proben dienten als Kontrolle (Proben mit nachgewiesener HPV16-DNA-Clearance), 72 Proben enthielten persistierende HPV-DNA ohne CIN2+ und 109 mit CIN2+. In jeder Probe wurde der
Methylierungsgrad von 66 verschiedenen CpG-Dinukleotiden untersucht.

In den diagnostischen Proben hatten $88 \%$ der CpG-Stellen von Frauen mit CIN2+ einen signifikant höheren Methylierungsgrad im Vergleich zu Kontrollproben. Der größte Wert der „Area under the ROC curve" (AUROC) betrug 0,82 für das CpG-Dinukleotid $6.457 \mathrm{im}$ L1-Gen. Die diagnostische Sensitivität von $91 \%$ korrespondierte mit einer Spezifität von $60 \%$ für CIN2+. $17 \%$ der CpG-Stellen in den prädiagnostischen CIN2+-Proben (median drei Jahre vor Diagnose) hatten einen signifikant höheren Methylierungsgrad im Vergleich zu den Kontrollen. Die stärkste prädiagnostische CpG-Stelle war in diesen Fällen $6.367 \mathrm{im} \mathrm{L1-Gen} \mathrm{mit} \mathrm{einer} \mathrm{AUROC}$ von 0,76. Altersstratifizierte Analysen lassen den Schluss zu, dass Frauen über einem medianen Alter von 28 Jahren ein

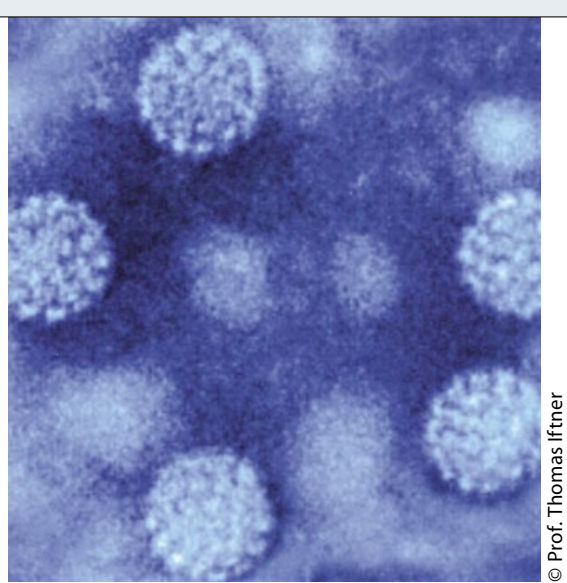

Der Methylierungsgrad im HPV-Genom könnte Prognosefaktor für CIN2+ sein.

erhöhtes Risiko für präkanzeröse Läsionen mit einem hohen Methylierungsgrad haben. Der höhere Methylierungsgrad in CIN2+-Proben lässt sich nicht mit einer höheren Viruslast erklären.

Fazit: Ein erhöhter Methylierungsgrad der HPV16-DNA in Zervix-Gewebeproben könnte möglicherweise als Prädiktor für ein aktuell vorliegendes und auch für ein sich erst noch entwickelndes CIN2+ herangezogen werden.

Barbara Kreutzkamp

Mirabello L et al. Elevated methylation of HPV16 DNA is associated with the development of high grade cervical intraepithelial neoplasia. Int J Cancer. 2013;132(6):1412-22.

\title{
Hoch dosierte interstitielle Brachytherapie bei fortgeschrittenem Zervixkarzinom
}

Eine konventionelle intrakavitäre Brachytherapie ist bei Patientinnen mit fortgeschrittenem Karzinom der Zervix oft nicht effektiv genug. Nun wurden Langzeitergebnisse zur interstitiellen Brachytherapie veröffentlicht.

$\mathrm{n}$ ihrer Studie wertete das Autorenteam
die Daten von 116 Patientinnen aus.
$91 \%$ der Frauen hatten Tumoren in den
Stadien IIB-IVA. Alle Teilnehmerinnen
erhielten eine externe Strahlentherapie
des Beckens mit einer medianen Dosis
von $50,4 \mathrm{G}$ in 28 Fraktionen.

Für die Planung der interstitiellen Brachytherapie (high-dose-rate interstitial brachytherapy, HDR-ISBT) errechnete ein spezielles Programm mithilfe vorliegender CT-Bilder die optimale Dosisverteilung. In Allgemeinnarkose oder unter epiduraler Anästhesie implantierten die Ärzte Hohlnadeln, die im Afterloading-Verfahren mit ${ }^{192}$ Iridium-Quel- len bestückt wurden. Die erste HDRISBT mit drei Fraktionen zu 6 Gy im Abstand von mindestens sechs Stunden erfolgte ein bis zwei Wochen nach Beendigung der externen Strahlentherapie.

Diese Prozedur wurde nach zwei weiteren Wochen mit der gleichen Dosierung wiederholt, sodass eine Gesamtdosis von 36 Gy in sechs Fraktionen resultierte. $81 \%$ der Frauen erhielten gleichzeitig Cisplatin; bei 61 \% erfolgte zusätzlich eine lokale Radiofrequenz-Hyperthermie-Behandlung.

Nach einer Beobachtungszeit von 35 Monaten war eine lokoregionale Kontrolle - ohne Hinweis auf Malignom im
Bereich des Originaltumors durch klinische und bildgebende Untersuchung bei $85,3 \%$ der Patientinnen dokumentiert. Die Drei-Jahres-Raten für das krankheitsfreie Überleben in den Stadien IB, II, III und IVA lagen bei $59 \%$, $67 \%, 71 \%$ und $57 \%$. Für die gesamte Kohorte betrugen die 5-Jahres-Überlebensraten $60 \%$ (krankheitsfreies Überleben) und $44 \%$ (Gesamtüberleben).

Fazit: In der vorliegenden Studie ergab die Kombination aus externer Bestrahlung und Hoch-Dosis-Brachytherapie hohe Ansprech- und Überlebensraten. Die Autoren empfehlen diese Therapie für Patientinnen, bei denen intrakavitäre Therapien nicht erfolgversprechend sind.

Andreas Fischer

Pinn-Bingham M et al. Outcomes of high-doserate interstitial brachytherapy in the treatment of locally advanced cervical cancer: long-termresults. Int J Radiat Oncol Biol Phys. 2013;85(3):714-20. 